\title{
Étude du comportement d'un mur en Terre Armée : rôle de l'interface sol/armature dans la modélisation
}

E. BOURGEOIS ${ }^{1}$

${ }^{1}$ Université Paris-Est

Laboratoire central des Ponts et Chaussées 58 , boulevard Lefebvre 75732 Paris Cedex 15

${ }^{2} \mathrm{SNCF}$

Direction de l'Ingénierie 6 , avenue

François-Mitterrand 93574 La Plaine-

Saint-Denis Cedex laurent.soyez@lcpc.fr emmanuel.bourgeois@lcpc.fr
Le LCPC a organisé et participé à un concours de prévision du comportement d'un ouvrage en Terre Armée dans le cadre des Journées nationales de géotechnique et de géologie de l'ingénieur en 2008. On présente deux modélisations numériques par éléments finis effectuées par le LCPC pour répondre à ce concours : la première a été réalisée à partir de motifs prédéfinis et la deuxième utilise un modèle homogénéisé. Dans un premier temps, on compare les résultats des calculs effectués avant les mesures, entre eux et avec les données expérimentales. On a ensuite cherché, pour les deux modélisations, à estimer l'influence des caractéristiques mécaniques du sol et de l'interface sur le comportement de l'ouvrage. On a également essayé de recaler a posteriori différents paramètres sur la base des mesures, notamment sur la base d'essais d'extraction d'armatures dans l'ouvrage expérimental. Une fois parvenu à un calage jugé satisfaisant, on a utilisé les modèles pour simuler la réponse du mur à un nouveau chargement statique vertical plus élevé que celui appliqué pour le concours.

Mots-clés : Terre Armée, modélisation numérique, interaction sol/armature, modèle homogénéisé, mesures expérimentales.

\section{Study of the behaviour of a Reinforced Earth wall: influence of the soil/reinforcement interface in modelling}

The results of a benchmark exercise concerning the behaviour of a Reinforced Earth wall were presented during the JNGG'08. This paper gives more details about two numerical models proposed by the French Public Works Research Laboratory (LCPC): the first one uses predefined patterns and the second one resorts to a homogenized model. First, predictions of both models are compared to experimental measurements. Then, the influence of geotechnical properties of soil and soil/reinforcement on the behaviour of the retaining wall is discussed, and new sets of values of the mechanical properties have been chosen in order to get a better agreement between simulations and measurements. Pullout tests have been carried out to improve the interface simulation. After that, both models were used to predict the response of the Reinforced Earth wall subjected to a larger loading. Numerical results are compared to experimental measurements.

Key words: reinforced earth, numerical modelling, soil/reinforcement interaction, homogenized model, experimental measurements. 


\section{Introduction}

Le LCPC et la SNCF ont lancé une collaboration portant sur l'utilisation de murs en Terre Armée pour des ouvrages ferroviaires, articulée autour du travail de thèse de Laurent Soyez. Un mur expérimental de $8 \mathrm{~m}$ de long et $3,5 \mathrm{~m}$ de haut a été construit au Centre d'expérimentation routière de Rouen (CER). Les armatures ont été équipées de jauges de contraintes, et le mur a été soumis à des chargements statiques et dynamiques. Un grand nombre de cycles ont été effectués, de manière à étudier le comportement à long terme du mur.

Dans un premier temps, on s'est intéressé aux déplacements du mur et aux contraintes dans les armatures lors d'un chargement statique. Cette étude a fait l'objet d'un concours de prévision dont les résultats ont été présentés lors des Journées nationales de géotechnique et de géologie de l’ingénieur à Nantes en juin 2008 .

Les données du problème et les chargements sont récapitulés dans Soyez et Le Kouby (2009).

Le LCPC a proposé plusieurs réponses reposant sur des simulations numériques par éléments finis, avec le logiciel CESAR-LCPC :

- une modélisation bidimensionnelle en déformation plane, dans laquelle les armatures des cinq lits du mur sont discrétisées par des éléments de massif ;

- des modélisations bidimensionnelles et tridimensionnelles reposant sur l'utilisation de modèles homogénéisés.

On présente tout d'abord les résultats des calculs prévisionnels effectués avec ces deux approches pour le concours de prévision, puis les résultats des tentatives de recalage des modèles faites après avoir eu connaissance des mesures effectuées sur le mur.

A partir de ces calages, on comparera les prévisions des modèles avec de nouvelles mesures réalisées en soumettant le mur à un chargement plus important que le précédent.

\section{2}

\section{Modélisation mécanique des murs en Terre Armée}

La méthode des éléments finis n'est pas utilisée comme outil de dimensionnement courant pour les murs en Terre Armée. Cependant, cette méthode, souple et polyvalente, permet d'analyser une grande variété d'ouvrages pouvant présenter une géométrie complexe, en tenant compte des propriétés mécaniques des différents matériaux et de phénomènes variés. De très nombreux ouvrages de géotechnique sont calculés sous l'hypothèse des déformations planes : c'est le cas des remblais, des murs de soutènement, et aussi d'ouvrages dont la géométrie réelle à la fin ou au cours de la construction ne respecte pas cette hypothèse (on peut penser par exemple aux ouvrages dont la construction implique un phasage complexe comme les tunnels, ou les parois moulées).

Dans le cas des murs en Terre Armée, la disposition des armatures dans le sol rend la structure étudiée nettement tridimensionnelle. Néanmoins, il reste cou- rant de procéder à des analyses en déformation plane (Bastick, 1987 ; Rospars et al., 2004 par exemple), les modélisations tridimensionnelles restant rares à cause de la lourdeur de la mise en œuvre d'un calcul dans lequel on discrétise chaque armature (Sellali-Haraigue, 1999). Dans le cas particulier du mur expérimental construit au CER, la nature du chargement appliqué à une traverse renforce le caractère tridimensionnel du problème.

Pour le concours de prévisions, le LCPC a effectué des simulations de deux types:

des calculs en déformations planes, avec une discrétisation des armatures de chaque lit;

- des calculs en conditions bidimensionnelle et tridimensionnelle, avec une représentation du sol renforcé par un modèle homogénéisé développé à l'ENPC et implanté dans une version recherche de CESARLCPC.

\section{git}

\section{Modèles discrets}

La modélisation des murs en Terre Armée pose différents problèmes bien spécifiques liés au fonctionnement des différentes parties de l'ouvrage et à son mode de construction. On présente ici les principaux choix de modélisation retenus.

Les écailles qui constituent le parement ne sont pas mécaniquement solidaires : elles ne constituent pas une coque continue susceptible de reprendre des efforts de flexion significatifs. On peut prendre en compte au moins partiellement (dans la direction verticale) cette caractéristique en introduisant dans le maillage un " joint » entre les écailles, auquel on attribue des paramètres élastiques réduits : le parement se comporte alors comme une succession de panneaux articulés entre eux par une liaison qui ressemble à une rotule. Les joints réels placés entre les écailles sont discontinus et mesurent quelques millimètres : pour des raisons pratiques de construction du maillage, les éléments finis qui les représentent ont une épaisseur fictive largement supérieure.

Le sol est considéré comme un milieu continu élastique - parfaitement plastique et son comportement est représenté par le modèle de Mohr-Coulomb. On a préféré un modèle simple : la mise en œuvre de modèles de comportements complexes donne souvent un sentiment trompeur de sophistication, alors que certains paramètres importants, comme la saturation du sol, sont mal connus, variables dans le temps, et peuvent avoir une influence plus significative que le raffinement apporté par une surface de charge ou une loi d'écrouissage complexes.

Le principe de fonctionnement des murs en Terre Armée repose sur le fait que le sol, en se déformant, transmet, par frottement, un effort longitudinal aux armatures. Une technique possible consiste à utiliser les « motifs prédéfinis » disponibles dans le préprocesseur MAX2D de CESAR-LCPC. Ces motifs comportent des éléments de massif (quadratiques), représentant les écailles, le sol, les armatures, et le joint entre écailles. Les armatures sont simulées par des éléments de faible épaisseur, et le contact avec le sol est décrit au moyen d'éléments de (c contact ») sans épaisseur (Richer, 1985), permettant de contrôler le frottement maximum entre le sol et l'interface. Deux couches d'éléments de contact 
sont nécessaires, une au-dessus et une au-dessous de l'armature.

Si les éléments d'interface ont un comportement de type Mohr-Coulomb, la contrainte normale sur les armatures contrôle le frottement maximal qui s'exerce sur l'armature : il est donc important que la contrainte verticale soit bien calculée. Or, lorsque des déformations plastiques importantes se produisent, une dilatance plastique significative peut augmenter le frottement entre le sol et les armatures. Ce phénomène de « dilatance empêchée » est pris en compte dans les méthodes de dimensionnement classiques des murs en Terre Armée (NF P 94-220-0), mais peut s'avérer difficile à maîtriser dans les calculs.

Par ailleurs, la technique consistant à associer à chaque lit d'armatures deux couches d'éléments de contact présente un inconvénient important : la plaque équivalente tend à séparer le sol en deux compartiments qui ( glissent » l'un sur l'autre. La cinématique calculée est alors discontinue de part et d'autre de la plaque, alors que le déplacement du sol dans le mur réel est continu. Cette difficulté est surmontée ici en ajoutant des éléments ad hoc, dits de relation linéaire (Unterreiner et al., 1997 ; Benhamida, 1998) entre les nouds (côté sol) situés en dessus et en dessous de chaque armatures.

Dans une première approche, les motifs prédéfinis ont été retenus car ils simplifient significativement la construction du maillage. Par contre, n'ayant pas de jeu de paramètres pour les éléments de contact, ceux-ci n'ont pas été pris en compte dans ce premier modèle 2D : la modélisation du frottement sol-armature se limite donc à un contact adhérent.

\section{9.}

\section{Modèles homogénéisés}

Compte tenu du nombre d'armatures placées dans le mur, et de leur disposition spatiale régulière, il est naturel de représenter le comportement global du mur en remplaçant la zone renforcée par les inclusions par un matériau ( homogène » équivalent, d'autant que, comme on l'a vu, les modélisations discrètes posent différents problèmes (introduction d'une « plaque équivalente » continue, alors que les armatures sont espacées dans la direction parallèle au mur ; difficulté de prendre en compte le caractère tridimensionnel du chargement appliqué sur une traverse). Par comparaison, un modèle homogénéisé simplifie fortement la réalisation d'un calcul tridimensionnel et permet donc de prendre en compte la géométrie réelle du chargement.

Une équipe de l'ENPC a développé depuis plusieurs années une famille de modèles visant à représenter le comportement des ouvrages renforcés par des inclusions. La démarche a commencé par l'étude des propriétés de résistance à l'échelle macroscopique du milieu renforcé (de Buhan, 1986), réinterprétées comme un critère de plasticité (Greuell, 1993), et a conduit ensuite à une modélisation mécanique élastoplastique complète (Sudret et de Buhan, 2001 ; Bennis et de Buhan, 2003). Le matériau hétérogène constitué par le sol et les inclusions est considéré comme un milieu homogène formé par la superposition de deux milieux continus, animés de cinématiques distinctes, et dans lesquels règnent des champs d'efforts intérieurs régis par des lois de comportement indépendantes. Géométriquement, les inclusions ne sont plus localisées, mais ( diluées ) dans le domaine occupé par le sol renforcé. Admettant que les inclusions travaillent essentiellement en traction (ou en traction-compression), on attribue au milieu continu représentant les inclusions un comportement élastoplastique unidimensionnel : les efforts intérieurs sont représentés par des scalaires. Le modèle obtenu est appelé « modèle multiphasique $)$.

On obtient une simplification significative du modèle en supposant que les deux milieux ont en fait la même cinématique (c'est-à-dire que l'on néglige le glissement du sol autour des armatures) mais en conservant deux descriptions distinctes des efforts intérieurs : le modèle correspondant est appelé « modèle adhérent ». Ce modèle simplifié présente l'inconvénient de conduire à des efforts non nuls aux extrémités des armatures. Pour cette raison, on a utilisé la formulation générale du modèle multiphasique, qui permet de mieux prendre en compte le transfert des efforts entre le sol et l'inclusion pour deux raisons

- elle introduit un paramètre de raideur appelé «coefficient d'interaction » qui traduit un effet d'échelle entre le diamètre des inclusions et leur espacement dans la structure;

- elle permet d'imposer une limite au frottement transmis par unité de longueur, moyennant une description appropriée de l'interaction sol-renforcement.

\section{8}

\section{Principaux choix de modélisation pour le mur de Rouen}

Pour le concours de prévision, on a effectué des calculs avec un modèle discret et des calculs avec le modèle homogénéisé. Bien que le chargement, appliqué à une traverse, ne permette pas de justifier une approche en déformation plane, on a effectué avec un modèle discret un calcul bidimensionnel, en répartissant la charge appliquée à la traverse sur une longueur fixée à 1,5 m, en considérant que la charge apportée sur un essieu se répartissait presque intégralement sur trois traverses successives.

Le maillage est constitué en utilisant des motifs prédéfinis dans le préprocesseur MAX2D de CESARLCPC. Le maillage du mur comporte cinq motifs de $14 \mathrm{~cm}$ d'épaisseur et $75 \mathrm{~cm}$ de hauteur, correspondant chacun à une demi-écaille. Les motifs incluent la possibilité de modéliser un joint entre deux écailles successives.

La construction de l'ouvrage est simulée en enchâ̂nant plusieurs étapes de remblaiement, de mise en place des armatures et/ou des écailles. Le processus comporte en tout sept étapes de calcul, au cours desquelles sont activés successivement les différents groupes d'éléments en prenant en compte leur poids volumique et leur comportement.

Après la fin de la construction, on applique une charge statique verticale de $90 \mathrm{kN}$ sur la traverse.

Le maillage utilisé est représenté sur la figure 1. Il comporte 6000 nœuds et 2200 éléments quadratiques. On ne représente pas le massif sous-jacent, et les conditions aux limites consistent à bloquer les deux composantes du déplacement sur la base du modèle. 
Avec les modèles homogénéisés, on a effectué des calculs bidimensionnels et tridimensionnels. Il n'y a pas de prise en compte du joint entre deux écailles adjacentes. Le modèle permet de ne pas reproduire le phasage de construction réel du mur, qui reflète la position des armatures. On simule la construction du mur en enchaînant cinq étapes au cours desquelles sont activés successivement les groupes d'éléments associés aux différentes couches; on applique le poids des groupes activés à chaque étape et on affecte des propriétés mécaniques à ces éléments.

Pour le contact entre les écailles et le massif de sol renforcé, on a introduit une couche d'éléments de faible épaisseur ( $5 \mathrm{~mm}$ ), auxquels on attribue un comportement élastique linéaire avec un module de compression élevé mais un module de cisaillement très faible (ce qui conduit à prendre un coefficient de Poisson voisin de 0,499, pour un module d'Young de $500 \mathrm{kPa}$ ), afin d'obtenir un contact « lisse ». Dans le calcul tridimensionnel, on a opté pour une solution plus simple, en assurant un contact lisse au moyen d'éléments spéciaux sans épaisseur (éléments de relation linéaire dans CESAR-LCPC).
Le modèle utilisé pour les calculs bidimensionnels est représenté sur la figure 2. Il comporte 5100 nouds et 1900 éléments quadratiques.

Le calcul en condition tridimensionnelle permet de prendre en compte (dans le maillage) la largeur réelle de la traverse : il n'y a donc pas besoin de faire des hypothèses a priori sur la diffusion de la charge en profondeur. Le maillage, représenté sur la figure 3, est moins fin que dans le cas bidimensionnel : il comporte 24000 nœuds et 5900 éléments quadratiques. Les conditions aux limites sont identiques à celles du calcul bidimensionnel : on ajoute des conditions de contact lisse sur les deux faces verticales parallèles qui limitent le maillage.

Le calcul est réalisé en supposant que les inclusions restent élastiques linéaires. Les paramètres à fournir sont le module du matériau qui les constitue $(210000 \mathrm{MPa})$ et la fraction volumique des armatures $\eta$ calculée comme la somme des aires de leurs sections divisée par la surface totale du mur :

$$
\eta=50 \frac{\mathrm{S}}{\mathrm{Lh}}=\frac{50 \cdot 0,045 \cdot 0,05}{3,75 \cdot 7,7}=4 \cdot 10^{4}
$$

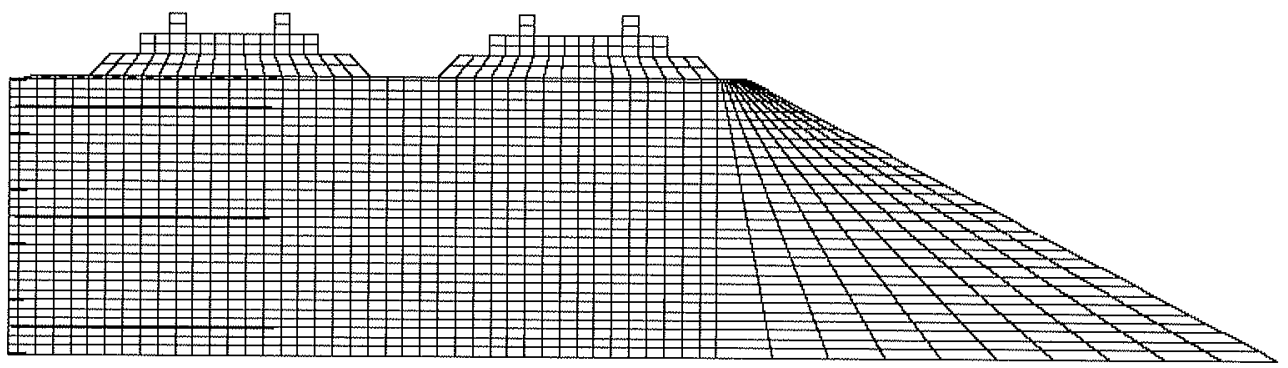

f6. 1 Maillage utilisé pour les modélisations en déformation plane avec le modèle discret. Mesh for the simulations with the discrete model (plane strain analysis).

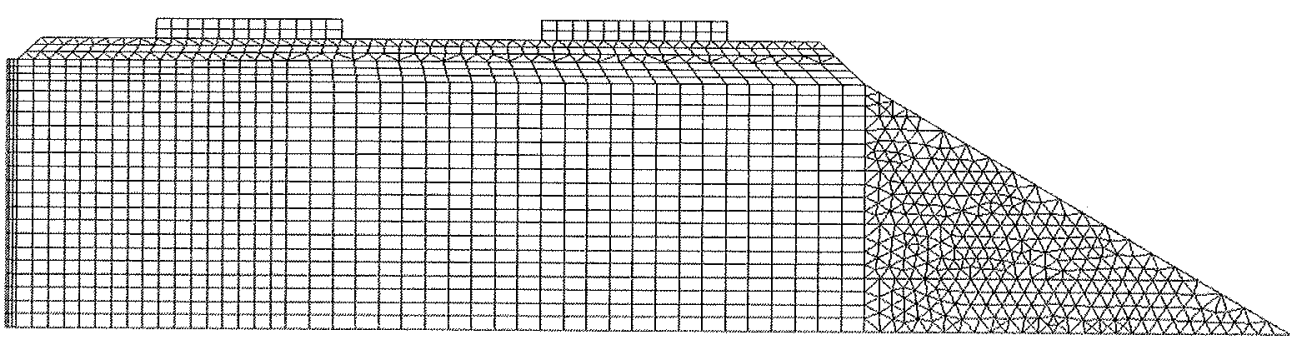

ศ6. 2 Maillage utilisé pour les modélisations en déformation plane avec le modèle multiphasique. Mesh for the simulations with the multiphase model (plane strain analysis).

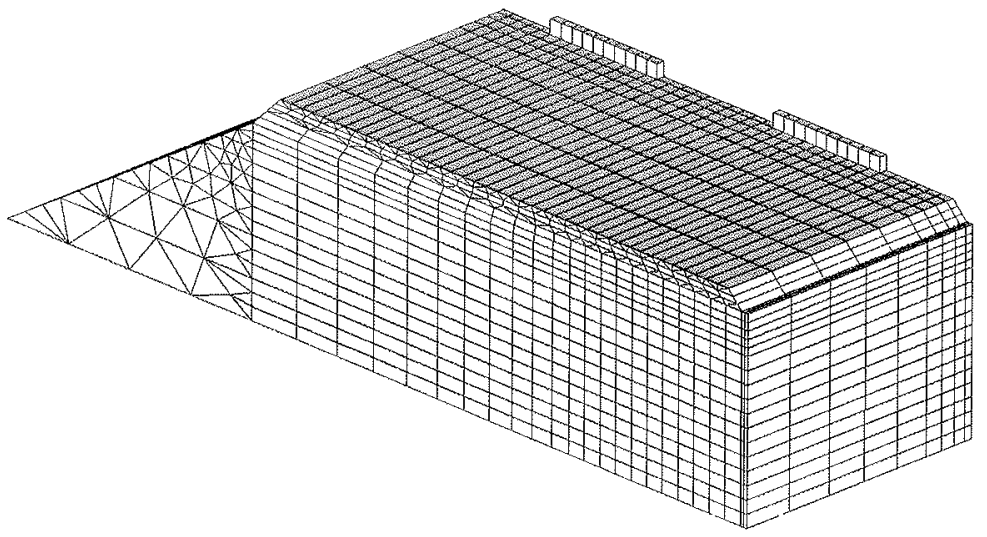

fi6.3. Maillage utilisé pour le calcul tridimensionnel (modèle multiphasique). Mesh for the three-dimensional simulations with the multiphase model. 


\begin{tabular}{|c|c|c|c|c|c|c|c|}
\hline \multirow[t]{2}{*}{ Tsisut } & \multicolumn{7}{|c|}{$\begin{array}{l}\text { Valeurs des paramètres mécaniques. } \\
\text { Mechanical properties values. }\end{array}$} \\
\hline & Wodere & 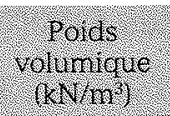 & 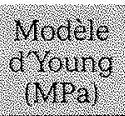 & 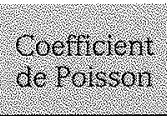 & 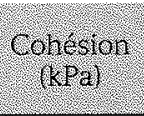 & 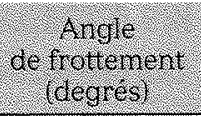 & 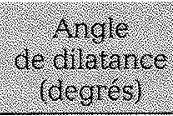 \\
\hline Écailles & élastique & 25 & 2000 & 0,25 & & & \\
\hline $\begin{array}{c}\text { Contact } \\
\text { écaille/remblai }\end{array}$ & élastique & 0 & 0,5 & 0,499 & & & \\
\hline Remblai & Mohr-Coulomb & 20,85 & 50 & 0,3 & 10 & 36 & 6 \\
\hline Grave & Mohr-Coulomb & 23,31 & 90 & 0,3 & 10 & 36 & 6 \\
\hline Ballast & élastique & 17 & 150 & 0,2 & & & \\
\hline
\end{tabular}

Le modèle multiphasique général introduit des paramètres supplémentaires concernant la raideur associée à l'interaction et la valeur du frottement maximal entre le sol et les inclusions. Par souci de simplicité, on a choisi, pour les calculs prévisionnels, de ne pas imposer de limite au frottement. On a choisi pour le coefficient d'interaction $\mathrm{c}_{\mathrm{l}}$ la valeur $7.10^{7} \mathrm{kPa} / \mathrm{m}^{2}$.

Le comportement du massif est représenté par une élasticité linéaire associée au modèle de DruckerPrager, avec les paramètres donnés dans le tableau I (données du concours de prévision).

\section{3}

\section{Comparaison des calculs de prévision entre eux et avec les mesures}

En complément des incréments de traction mesurés dans les armatures lors de l'application de la surcharge $(90 \mathrm{kN})$, on utilise aussi les mesures faites en fin de construction pour valider les modèles, bien qu'elles n'aient pas été communiquées aux participants du concours.

\section{3.}

\section{Comparaison des résultats obtenus avec les modèles 2D}

Dans un premier temps, on a comparé les résultats obtenus avec les deux modèles 2D avant de s'intéresser aux mesures. Les déplacements verticaux de l'interface remblai/sous-couche sont en moyenne de l'ordre de $0,7 \mathrm{~mm}$ dans les deux cas. Les écarts entre les valeurs calculées par les deux modèles sont inférieurs à $0,02 \mathrm{~mm}$.

Les résultats des calculs d'incrément de traction dans les armatures sont proches. On note toutefois deux différences. La première réside dans l'allure des courbes à proximité du parement. Le modèle homogénéisé fait apparaître un point d'inflexion pour les trois lits, ce qui n'est pas le cas du modèle discret. Deuxièmement, les incréments de traction aux bouts libres des armatures ne sont pas nuls avec le modèle discret.

Les déplacements horizontaux du parement reflètent les différences entre les modélisations du parement, considéré comme continu dans le calcul avec le modèle multiphasique, alors que dans le modèle discret, les joints entre écailles sont pris en compte explicitement au moyen d'éléments plus souples.
Les résultats des calculs étant similaires, seuls ceux du modèle homogénéisé sont présentés dans la suite de cette partie.

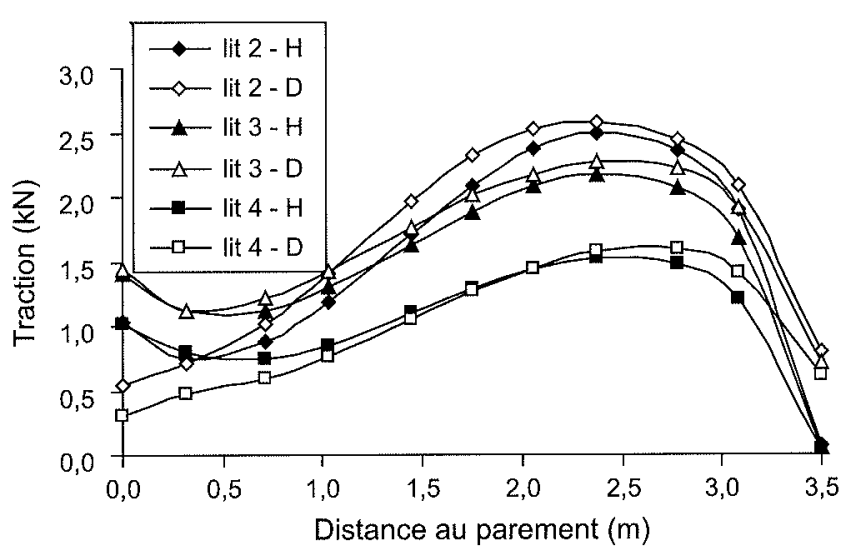

16. 4 Comparaison des variations de traction liées à l'application de la surcharge calculées dans les lits 2-3-4 avec les modèles 2D (H : homogénéisé ; D : discret).

Comparison of the increase of tensile forces due to loading in strip levels 2-3-4 for 2D models ( $\mathrm{H}$ : homogenized; $\mathrm{D}$ : discrete).

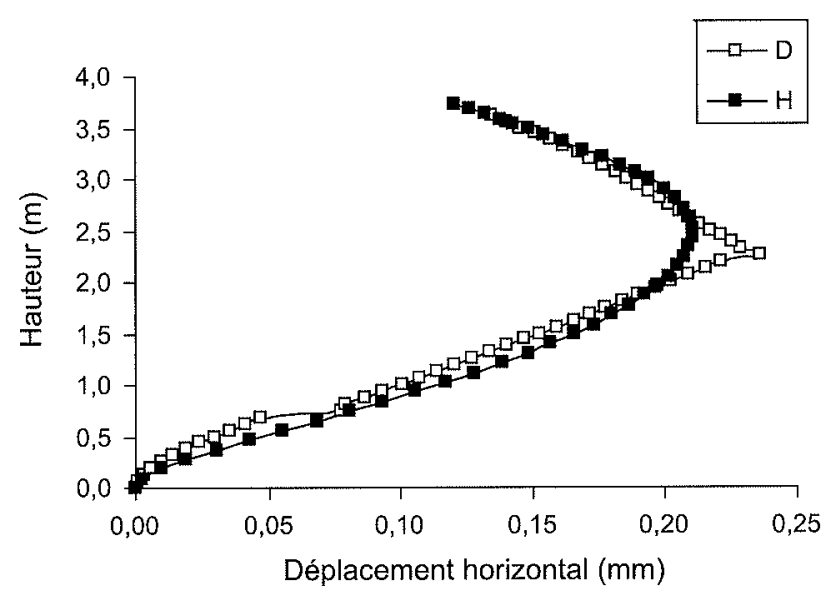

HiG. \& Déplacements horizontaux du parement liés à l'application de charge calculés avec les modèles $2 \mathrm{D}(\mathrm{H}$ : homogénéisé ; D : discret).

Facing horizontal displacements due to loading for 2D model ( $\mathrm{H}$ : homogenized ; D : discrete). 


\section{Efforts dans les armatures à la fin de la construction}

On s'intéresse dans un premier temps aux tractions calculées dans les armatures à la fin de la construction, pour les lits 2, 3 et 4 .

Les tractions calculées sont un peu faibles pour les lits 2 et 3 (Figs. 6 a et 6b). Les valeurs calculées pour le lit 4 sont proches des mesures (Fig. 6c). Les calculs pour le lit 5 (non représentés ici) prévoient des compressions dans les armatures.
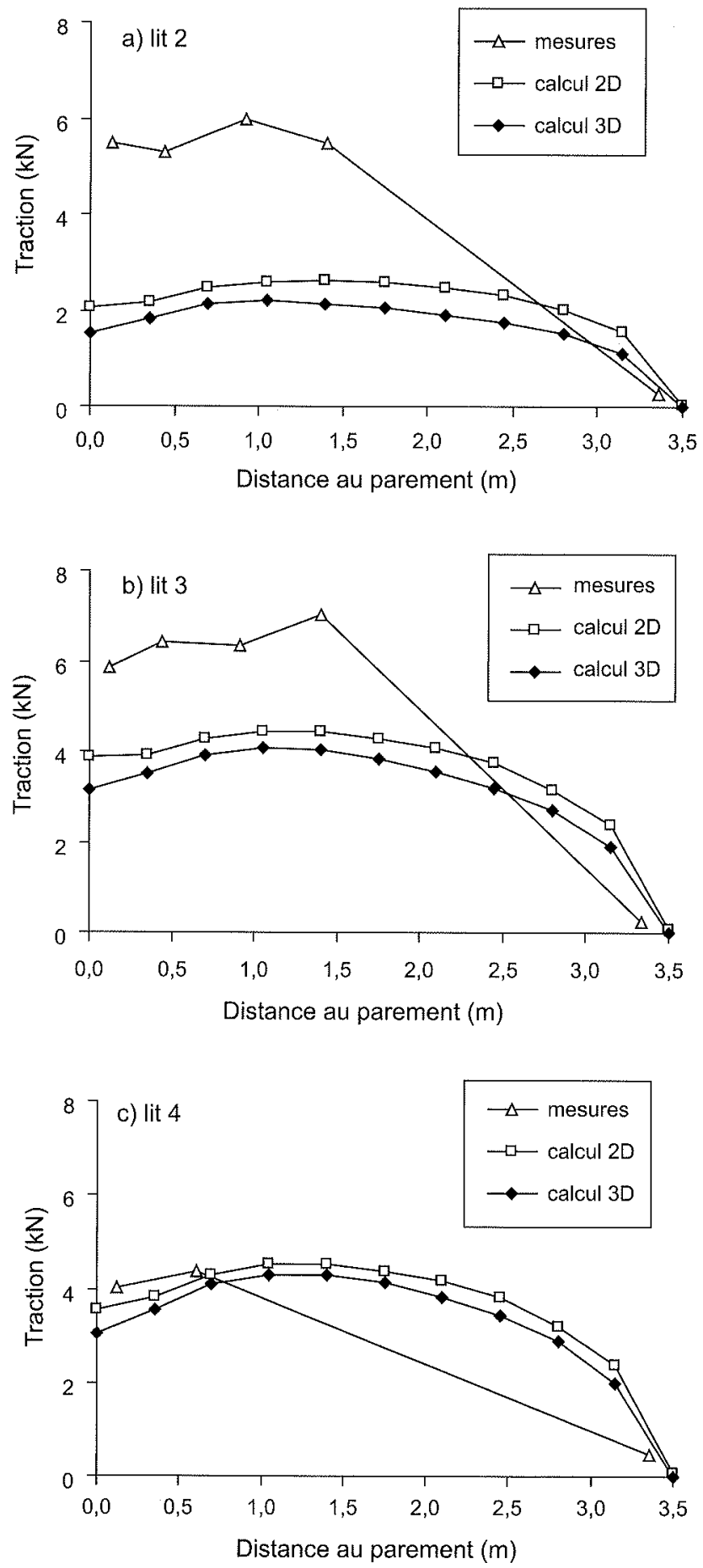

16. 6 Efforts dans les armatures des lits 2, 3 et 4 à la fin de la construction.

Tensile forces at the end of construction in the strip level 2, 3 and 4

\section{Variations des efforts dues à la surcharge}

Les figures $7 \mathrm{a}, \mathrm{b}, \mathrm{c}$ présentent les augmentations de traction dans les lits 2 à 4 calculées avec les modèles homogénéisés 2D et 3D, ainsi que les mesures.

Il apparaît clairement que les calculs prévisionnels surestiment largement ( $d^{\prime}$ un facteur parfois supérieur à 50) les augmentations de traction provoquées par l'application de la surcharge. On note également que la modélisation tridimensionnelle donne qualitativement des résultats moins mauvais.
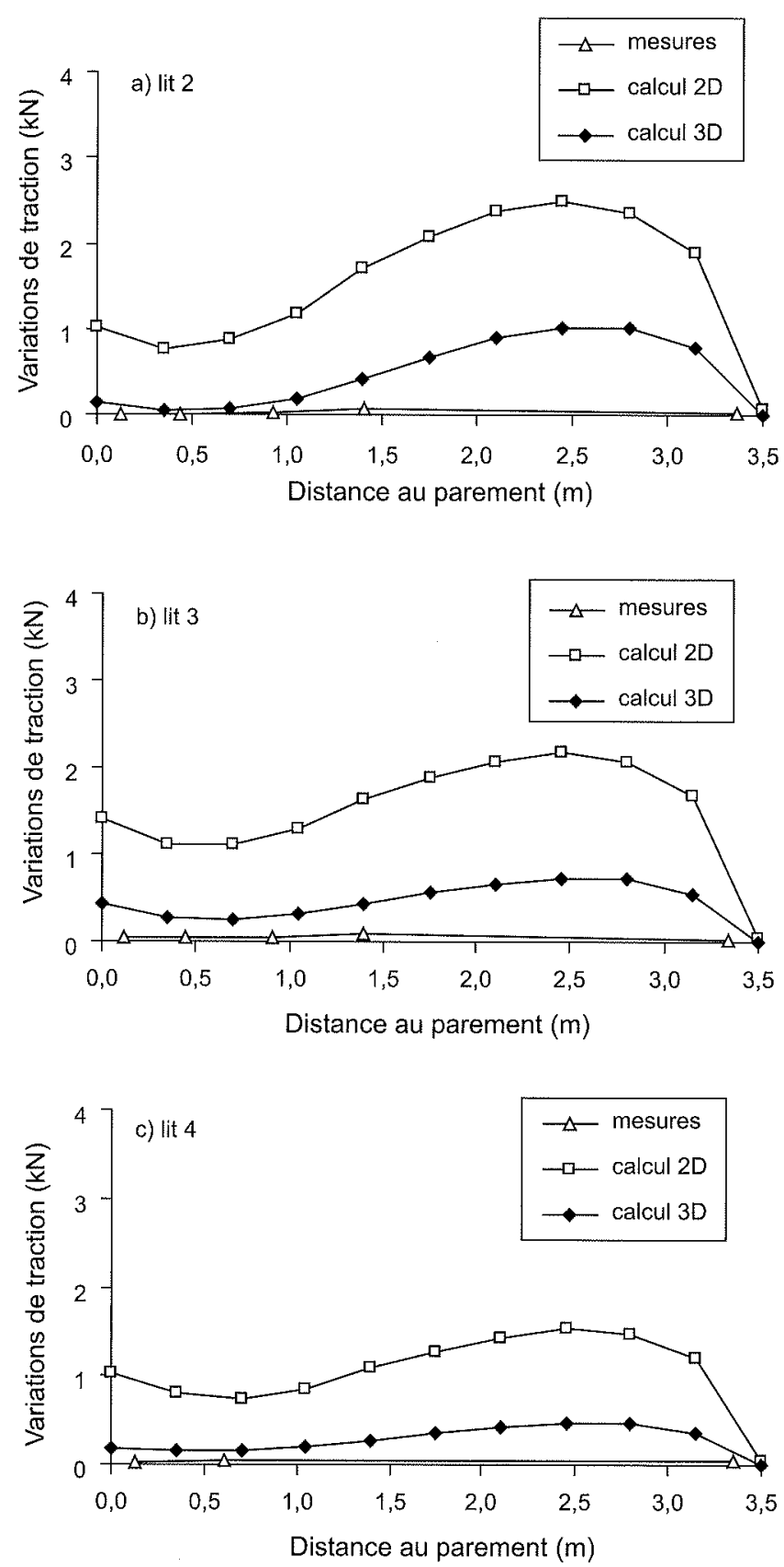

16. 7 Augmentations de traction dans les armatures des lits 2, 3 et 4 pour une charge de $90 \mathrm{kN}$ mesurées et calculées avec les modèles $2 \mathrm{D}$ et $3 \mathrm{D}$.

Increase of tensile forces due to the $90 \mathrm{kN}$ loading measured and computed for $2 \mathrm{D}$ and $3 \mathrm{D}$ models in the strip level 4. 


\section{Déplacements dus à la surcharge}

Le concours de prévision demandait aux participants d'évaluer, d'une part, les tassements en différents points situés à l'interface remblai/sous-couche et, d'autre part, le déplacement horizontal du parement.

Concernant les tassements, les mesures, comme les calculs, indiquent que l'application de la charge audessus du remblai renforcé $(x \in[2,25 ; 3,75])$ produit un tassement à peu près identique à celui obtenu lorsque l'on charge la deuxième traverse, plus éloignée du parement $(x \in[7,25 ; 8,75])$. Les mesures donnent des valeurs de l'ordre de $0,1 \mathrm{~mm}$; les calculs donnent des tassements de $0,75 \mathrm{~mm}$ et $0,5 \mathrm{~mm}$ respectivement en conditions bidimensionnelle et tridimensionnelle.

Il en va de même pour les déplacements horizontaux du parement. Les mesures indiquent des valeurs de 0,01 mm environ alors que les maxima obtenus par le calcul avoisinent $0,06 \mathrm{~mm}$ en $3 \mathrm{D}$ et $0,2 \mathrm{~mm}$ en $2 \mathrm{D}$. Néanmoins, les allures des courbes sont correctes : elles présentent un bombement du mur dans sa partie supérieure avec un déplacement maximal aux environs de $2,5 \mathrm{~m}$ (Fig. 8).

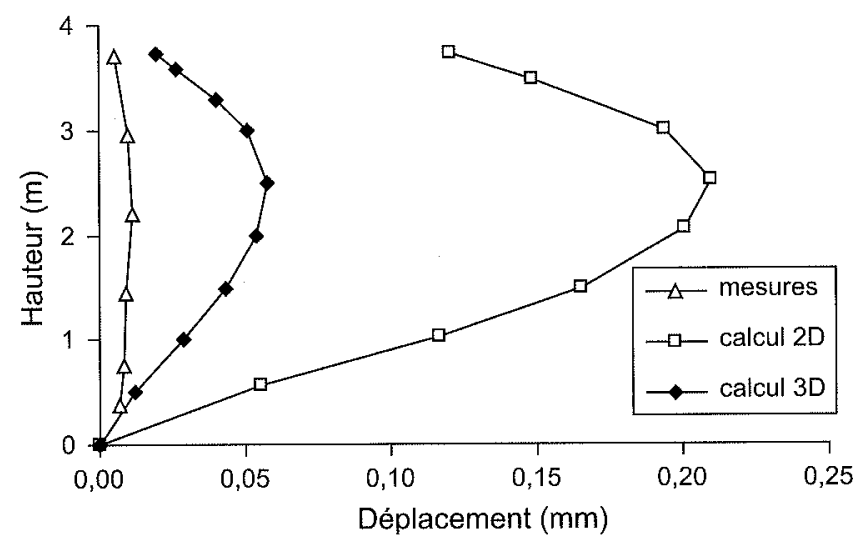

ria. 8 Déplacements horizontaux du parement (en $\mathrm{mm}$ ) pour une charge de $90 \mathrm{kN}$ mesurés et calculés avec les modèles $2 \mathrm{D}$ et $3 \mathrm{D}$.

Facing horizontal displacement (mm) due to the $90 \mathrm{kN}$ loading measured and computed for 2D and $3 \mathrm{D}$ models.

\section{3}

\section{Conclusions des calculs a priori}

De manière générale, les efforts calculés à la fin de la construction sont de l'ordre de 3 à $4 \mathrm{kN}$ au maximum. Ils sont donc inférieurs aux valeurs mesurées, dont le maximum varie entre 6 et $8 \mathrm{kN}$ selon les lits d'armatures. Compte tenu des différentes hypothèses faites, ces résultats sont raisonnables pour des calculs prévisionnels.

En revanche, les calculs surestiment largement les incréments de traction dans les armatures lorsque l'on charge la traverse la plus proche du mur. Les valeurs mesurées ne dépassent jamais $0,09 \mathrm{kN}$ : cette valeur est obtenue pour le lit 3 . Les calculs fournissent des valeurs allant jusqu'à $3 \mathrm{kN}$ dans les calculs bidimensionnels.
Pour le calcul tridimensionnel, on obtient pour le lit 3 un maximum de $0,7 \mathrm{kN}$, qui reste largement supérieur à la valeur mesurée. On vérifie que le problème présente un caractère tridimensionnel marqué, mal pris en compte par l'application dans un calcul 2D d'une force réduite, calculée en supposant que la charge se répartit uniformément sur une longueur de mur égale à l'intervalle entre deux traverses.

Les calculs surestiment aussi largement les déplacements horizontaux du parement et les tassements.

\section{4 \\ Recalage des modèles sur la base des mesures}

Une étude paramétrique concernant les caractéristiques mécaniques du matériau de remblai a été entreprise en 2D (modèle discret) pour déterminer leur influence sur le comportement global de l'ouvrage et diminuer l'écart entre les résultats calculés et mesurés.

Pour réduire les déplacements calculés, on a d'abord augmenté le module en prenant 250 puis $500 \mathrm{MPa}$. On diminue alors les déplacements (divisés par un facteur 5 ou 6), mais les tractions dans les armatures (en fin de construction) se trouvent aussi fortement diminuées. Ce n'est donc pas ce paramètre qui permet d'obtenir un bon recalage.

Le coefficient de Poisson du sol modifie la réponse de l'ouvrage : pour une valeur de 0,15 les déplacements liés à l'application de la charge sont diminués mais, comme pour le module d'Young, les tractions en fin de construction chutent fortement.

Diminuer la cohésion n'a pas d'impact significatif sur les déplacements verticaux mais augmente les déplacements du parement. Les tractions ne sont réellement modifiées que dans le premier mètre de l'armature.

Enfin, l'angle de frottement du sol n'a qu'une influence très limitée sur les résultats, pour les valeurs adoptées, et compte tenu des hypothèses et des choix de modélisations retenus.

On a tenté, pour le modèle 3D, d'obtenir des variations de traction plus faibles en considérant d'autres caractéristiques mécaniques dans la partie haute du modèle, pour modifier le moins possible les résultats à la fin de la construction. On a fait varier le module de la couche de GNT et les paramètres du sol constituant le remblai sans améliorer notablement les résultats.

En 2D, le seul moyen de diminuer l'impact du chargement sans diminuer les tractions dans les armatures en fin de construction consiste à prendre en compte différemment la diffusion de la charge dans la direction parallèle au parement. En admettant que celle-ci se diffuse suivant une répartition à 1 pour $1(\mathrm{H} / \mathrm{V})$, la variation de contrainte verticale au niveau du lit qui se trouve à mi-hauteur est environ 14 fois plus petite que celle directement sous la traverse. Cette valeur de surcharge fait passer l'augmentation maximale de traction dans le lit 3 de 2,3 kN à 0,6 kN (Fig. 9).

Les déplacements verticaux du parement diminuent environ d'un facteur 4 (Fig. 10). De même, les déplacements verticaux de l'interface sont ramenés d'environ $0,75 \mathrm{~mm}$ à $0,2 \mathrm{~mm}$. 


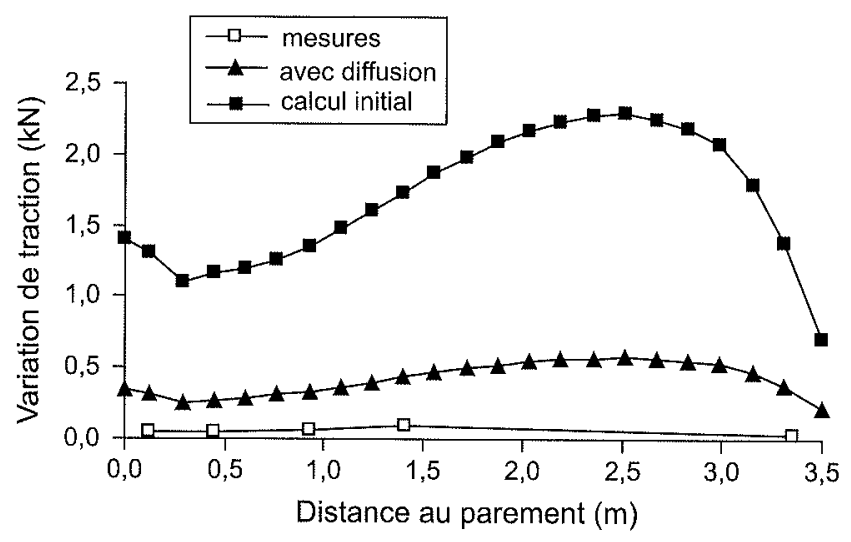

fa. 9 Variations des efforts dans les armatures du lit 3 pour une charge de $90 \mathrm{kN}$ mesurées et calculées avec deux modes de prise en compte de la surcharge.

Increase of tensile forces due to loading in strip level 3 measured and computed with two methods of conversion of the load in plane strain condition.

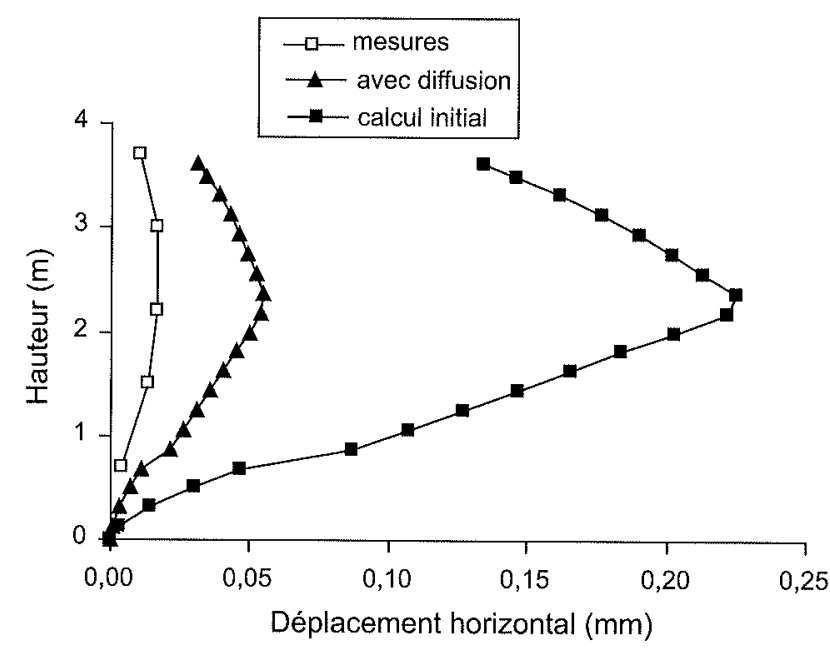

Ho od Déplacements horizontaux du parement pour une charge de $90 \mathrm{kN}$ mesurés et calculés avec deux modes de pris en compte de la surcharge.

Facing horizontal displacements due to the $90 \mathrm{kN}$ loading measured and computed with two methods of conversion of the load in plane strain condition.

\section{Travail sur le modèle d'interface sol/armature}

Un travail sur la modélisation de l'interface a ensuite été entrepris en parallèle sur les modèles 2D discret et 3D homogénéisé.

\section{5it. \\ Modèle discret 2D}

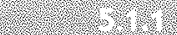 \\ Introduction d'une couche d'éléments fins autour des armatures et d'éléments de contact sol/écaille}

On a utilisé une couche d'éléments de massif de faible épaisseur autour de l'armature pour améliorer la modélisation de l'interface sol/armature (Fig. 11). Cette technique n'est pas compatible avec l'utilisation des motifs prédéfinis, ce qui a conduit à les abandonner.

On a attribué à ces éléments une loi de comportement de type Mohr-Coulomb et, dans un premier temps, les caractéristiques sont celles du sol. Ces éléments d'interface permettent d'améliorer légèrement les valeurs des tractions en fin de construction, et d'obtenir des valeurs en bouts des armatures comprises entre 0 et $-0,1 \mathrm{kN}$, donc nettement plus faibles en valeur absolue que celles obtenues avec le premier maillage.

Une étude de l'influence de l'angle de frottement de ces éléments a montré qu'une diminution de celuici entraîne une augmentation des tractions dans les armatures. Pour remédier à cette incohérence, on a été amené à prendre une cohésion et un angle de dilatance faibles.

Les murs en Terre Armée ont une particularité : le mur est construit par remblaiements successifs de couches de sol derrière les écailles. Compte tenu du contraste de raideur entre les écailles et le remblai, les couches de sol subissent sous le poids des couches sus-jacentes un tassement plus grand que celui des écailles. Cette différence justifie un traitement particulier pour l'interface sol-écaille : on a choisi d'utiliser des éléments sans épaisseur spécifiques (éléments de (c relation linéaire » dans CESAR-LCPC).

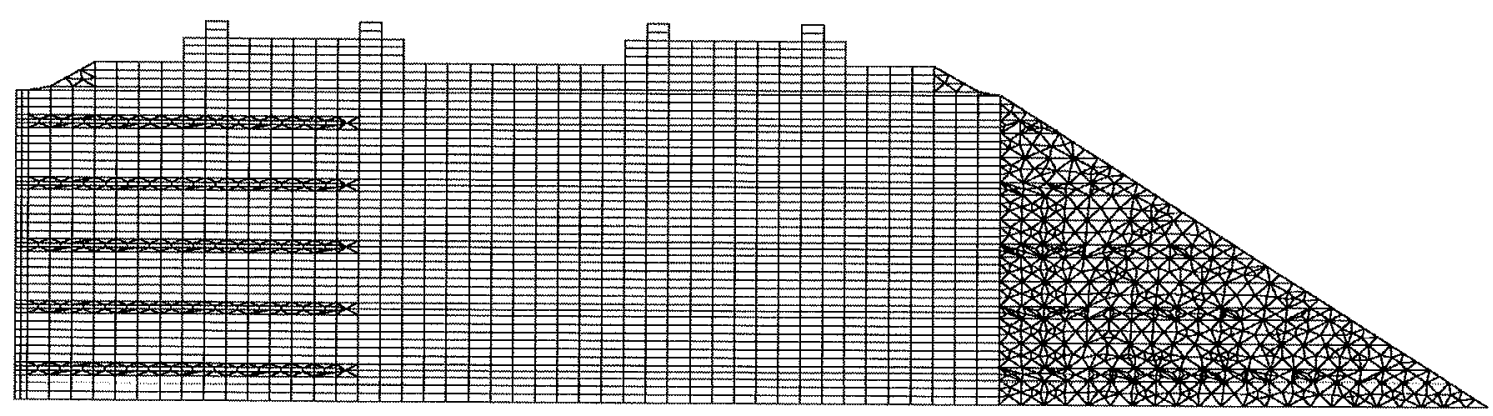

FG. 11 Maillage 2D discret avec une interface sol/armature de type élément de massif. 2D discrete mesh : soil/trip interface composed of volume elements. 


\section{0.}

\section{Détermination des paramètres au moyen d'essais d'extraction}

Suite aux premières tentatives de recalage, on a choisi de fixer à zéro les angles de frottement et de dilatance des éléments d'interface sol/armature. La contrainte de cisaillement est alors inférieure ou égale à la cohésion. Par ailleurs, on attribue aux éléments un module d'Young qui caractérise la raideur de l'interface.

Des essais d'extraction de trois armatures appartenant aux lits 1, 3 et 4 ont été menés sur le plot expérimental du CER (Soyez et Le Kouby, 2009). Deux méthodes ont été envisagées pour déterminer les paramètres des éléments d'interface à partir de ces essais. La première consiste en la réalisation d'un modèle numérique spécifique pour simuler ces essais. Le modèle correspond à une couche du massif en Terre Armée et comporte trois groupes d'éléments associés au matériau de remblai, à l'interface sol/armature et à l'armature. L'écaille n'est pas modélisée. Les déplacements horizontaux et verticaux sont bloqués à la base du massif. Les déplacements horizontaux des bords latéraux du remblai sont fixés à zéro. On applique une surcharge sur le bord supérieur du modèle correspondant au poids des couches de sol situées au-dessus de l'armature extraite. Enfin, une contrainte horizontale dirigée vers l'extérieur du modèle est appliquée au niveau de l'attache de l'armature pour simuler la force d'extraction exercée par le vérin. La modélisation de l'essai d'extraction permet de déterminer, d'une part, la cohésion à attribuer aux éléments d'interface (en ajustant la force maximale que l'on peut exercer sur l'armature dans le modèle), et, d'autre part, le module d'Young, en ajustant les déplacements (Fig. 12). Cette opération est répétée pour chacun des lits.

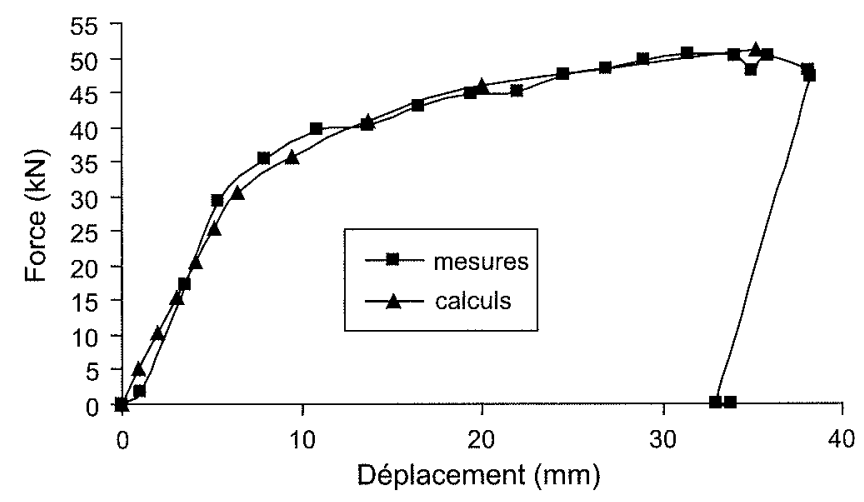

nc. 18 Calage de l'essai d'extraction du lit 3. Fit of the level 3 pull-out test.

Cette démarche conduit à la détermination de modules d'Young très faibles pour les éléments d'interface, de l'ordre de quelques $\mathrm{kPa}$. En introduisant ces valeurs dans le modèle global de l'ouvrage, les éléments d'interface induisent des tassements importants de l'ouvrage, de l'ordre de $10 \mathrm{~mm}$.

On a donc proposé une deuxième approche, analytique, pour choisir les paramètres des éléments représentant l'interface sol/armature. On considère que la pente de la courbe force/déplacement donne un coefficient de raideur dont la valeur est à relier au module $d^{\prime}$ Young. La cohésion équivalente est déterminée par la résistance au cisaillement que la couche représentant l'armature est capable de fournir. On obtient alors un jeu de paramètres pour l'interface de chaque lit d'armatures.

Les tractions dans les armatures en fin de construction se trouvent améliorées (Fig. 13). La réponse de l'ouvrage à l'application de la surcharge est sensiblement la même, aussi bien pour les incréments de traction dans les armatures que pour les déplacements.

On a entrepris une nouvelle fois de changer le module d'Young du matériau de remblai mais le constat est le même que lorsque les paramètres n'avaient pas été calés.

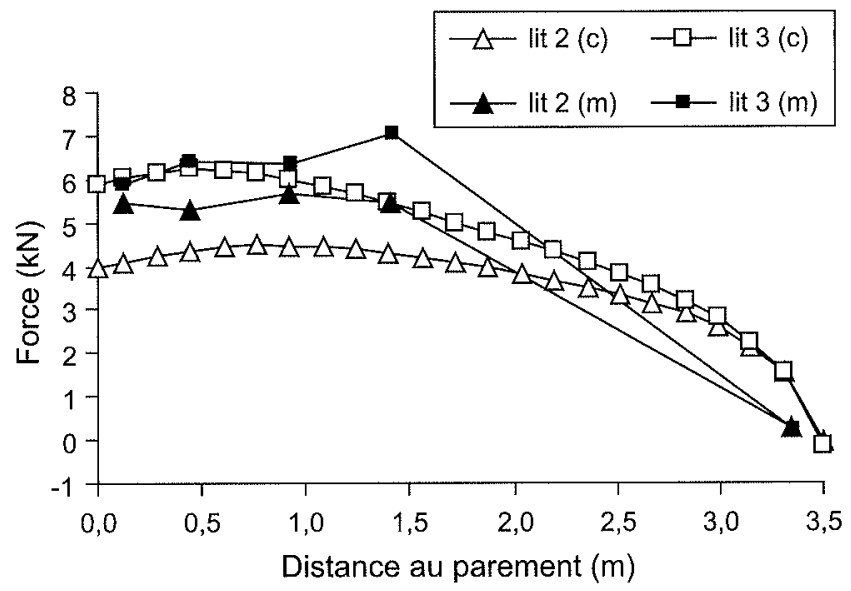

16. 13 Tractions mesurées (m) et calculées (c) en fin de construction dans les armatures des lits 2 et 3 .

Tensile forces measured $(\mathrm{m})$ and computed (c) in the strips levels 2 and 3 at the end of construction.

\section{Modèle homogénéisé 3D}

Différentes tentatives ont été effectuées pour améliorer les résultats des calculs bidimensionnels et tridimensionnels avec le modèle homogénéisé. On a choisi de recaler les résultats des calculs tridimensionnels.

\section{S.}

\section{Influence du coefficient d'interaction}

On s'est intéressé au paramètre d'interaction $\mathrm{C}_{i}$ qui caractérise, dans le modèle multiphasique, la raideur de l'interaction mécanique entre le sol et les armatures. Il a une influence directe sur les tractions calculées, mais ne permet pas de contrôler entièrement le résultat des simulations. On parvient, en ajustant ce paramètre, à des efforts calculés en fin de construction 2 à 3 fois plus faibles que ceux mesurés, et des variations 2 à 3 fois plus grandes que les valeurs mesurées, ce qui constitue un progrès par rapport à la situation précédente. Pour les tassements et le déplacement horizontal, les résultats sont remarquablement stables lorsque l'on fait varier $\mathrm{c}_{\mathrm{i}}$ (environ $0,5 \mathrm{~mm}$ pour le tassement et $0,06 \mathrm{~mm}$ pour le déplacement horizontal maximal). 


\section{(2)}

\section{Choix du frottement maximal sol/armatures}

Dans les calculs précédents, l'effort que le sol pouvait transmettre aux armatures par frottement n'était pas limité. Le modèle multiphasique permet cependant d'attribuer à l'interaction sol/armatures un comportement non linéaire : l'effort transmis aux armatures par le sol dépend linéairement du déplacement relatif entre les deux, jusqu'à un seuil sur l'effort d'interaction audelà duquel l'armature glisse librement. On note $I_{\max }$ la valeur maximale de la force d'interaction (il s'agit d'une force volumique, exprimée en $\mathrm{kPa} / \mathrm{m}$ ). On a choisi en première approche une valeur moyenne pour tout le massif, donnée par le produit de la surface des armatures par une contrainte de cisaillement de référence évaluée en fonction de la contrainte verticale moyenne et de l'angle de frottement du sol. Avec cette valeur $\left(\mathrm{I}_{\max }=11 \mathrm{kPa} / \mathrm{m}\right)$, les résultats sont pratiquement les mêmes que dans le cas précédent. On a alors réduit la valeur maximale de la force d'interaction à $5 \mathrm{kPa} / \mathrm{m}$. La figure 14 présente les efforts de traction calculés dans les différentes armatures. On note que pour les lits 2, 3 et 4, les courbes tendent vers une asymptote dans la partie droite du graphique, indiquant que le frottement maximal est mobilisé sur une partie de la longueur de l'armature. Dans ces zones, l'effort de traction est fixé par le frottement mobilisable : il en résulte que les incréments obtenus lorsqu'on applique la surcharge sur la traverse (Fig. 15) sont beaucoup plus faibles que dans les simulations précédentes et plus proches des mesures (sauf pour le premier lit, pour lequel il serait légitime d'utiliser un frottement maximal plus faible, compte tenu du fait que la contrainte normale est faible).

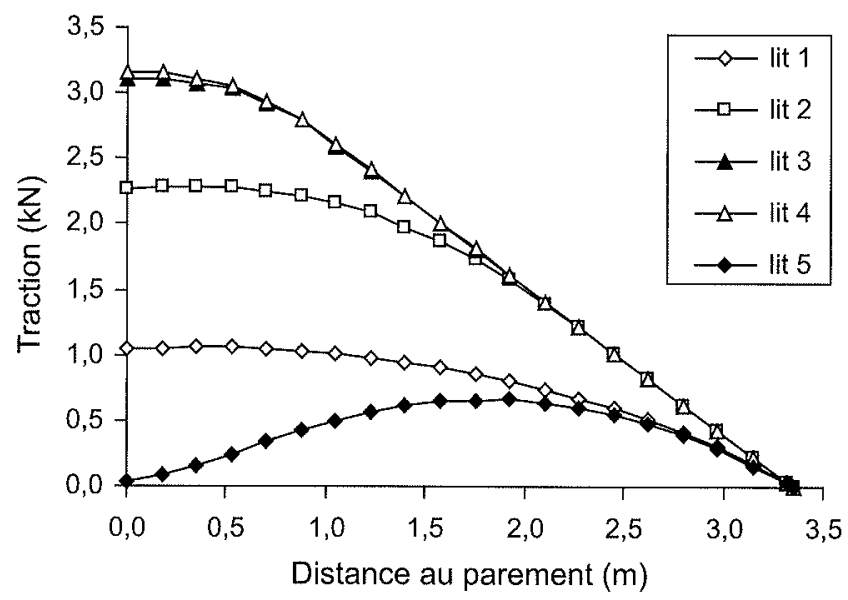

716. 14 Forces de traction dans les armatures des cinq lits à la fin de la construction : prise en compte d'une valeur maximale du frottement sol/armatures.

Tensile forces in the 5 strip levels : taking into account the maximal value of the soil/ reinforcement friction.

Avec cette approche, on parvient à des efforts en fin de construction à peu près deux fois inférieurs à ceux mesurés, et à des augmentations de force de l'ordre de ceux qui ont été mesurées. Sur le plan qualitatif, la répartition d'effort le long des armatures tend à devenir linéaire pour les lits 3 et 4, avec une valeur nulle au bout et une pente imposée par le paramètre de frottement maximal I max fourni dans les données du modèle.

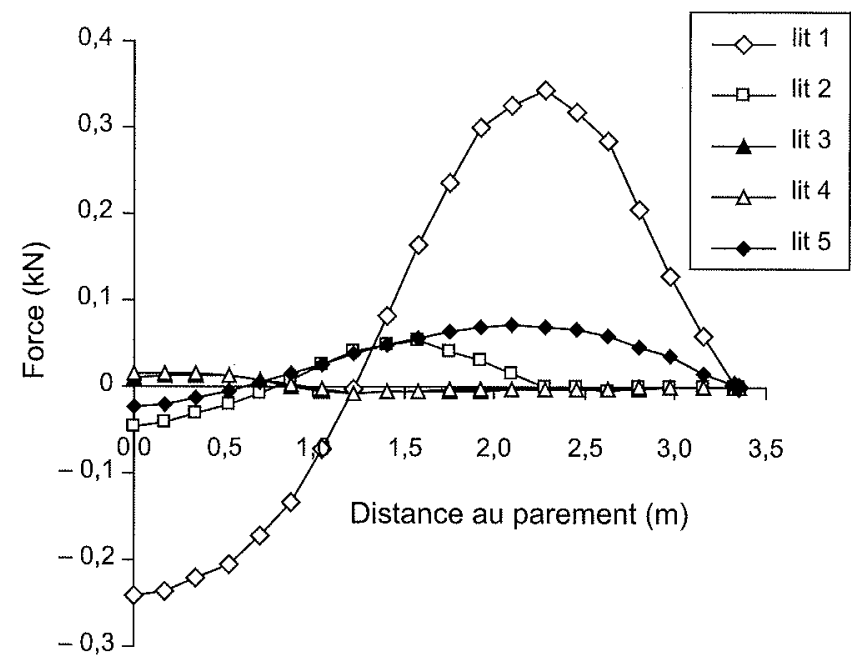

16. 15. Variations de traction dans les armatures, avec prise en compte d'une valeur maximale du frottement sol/armatures.

Increase of tensile forces in the 5 strip levels taking into account the maximal value of the soil/reinforcement friction.

Si on augmente la raideur d'interface $\mathrm{c}_{\mathrm{i}}$, on obtient un profil complètement linéaire pour ces lits à la fin de la construction : dans ce cas, le frottement étant complètement mobilisé le long des armatures à cette étape, la distribution des tractions n'évolue pratiquement pas dans les étapes de chargement ultérieures. Il y a donc une interaction entre le choix des valeurs des paramètres décrivant la raideur de l'interaction et le frottement limite : le second contrôle la valeur limite de la pente de la courbe de traction, et le premier contrôle la distance sur laquelle la traction varie linéairement.

A ce stade, on est parvenu à un bon calage des résultats calculés sur les mesures des tractions, mais les tassements calculés restent sensiblement plus grands que les valeurs mesurées. Pour l'ensemble des calculs précédents, le tassement maximal (à l'interface entre le remblai et la sous-couche) est sensiblement le même pour la partie renforcée et pour le remblai technique non renforcé, de l'ordre de $0,5 \mathrm{~mm}$, soit environ 5 fois plus que les valeurs mesurées.

L'essentiel du corps de remblai semblant être dans le domaine élastique, on peut proposer de tenir compte de la non-linéarité du comportement élastique des sols en augmentant le module du sol dans le domaine des petites déformations. On a donc multiplié le module d'Young par 3 (en s'inspirant des remarques faites au sujet de la modélisation des fondations superficielles de Labenne par Mestat et Berthelon, 2001). Cet ajustement diminue les tassements mais également les efforts calculés dans les armatures, ce que l'on peut corriger en augmentant la valeur du coefficient de Poisson. On a donc refait de nouvelles simulations avec $v=0,45$. Les tractions calculées pour ce dernier calage sont données par les figures 16 et 17 . On notera que les efforts dans les lits 2 et 3 sont sous-estimés tandis que les efforts dans le lit 4 sont surestimés. Les variations calculées sont dix fois plus faibles que dans les calculs prévisionnels, et de l'ordre des valeurs mesurées (même si les calculs donnent des valeurs un peu fortes pour le lit 2).

On obtient alors des tassements voisins de $0,2 \mathrm{~mm}$, et un déplacement horizontal du mur lors du chargement de la traverse de 0,04 $\mathrm{mm}$. Ces valeurs sont un peu plus élevées, mais du même ordre que les valeurs mesurées. 


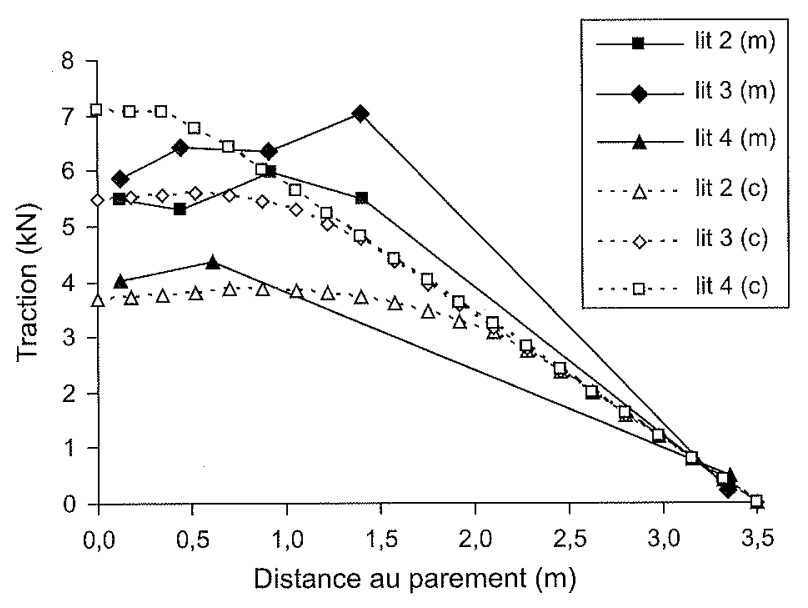

FI. 16 Tractions mesurées (m) et calculées (c) dans les armatures à la fin de la construction $(E=150 \mathrm{MPa}, v=0,45)$.

Tensile forces measured $(\mathrm{m})$ and computed $(\mathrm{c})$ at the end of construction $(E=150 \mathrm{MPa}, v=0,45)$.

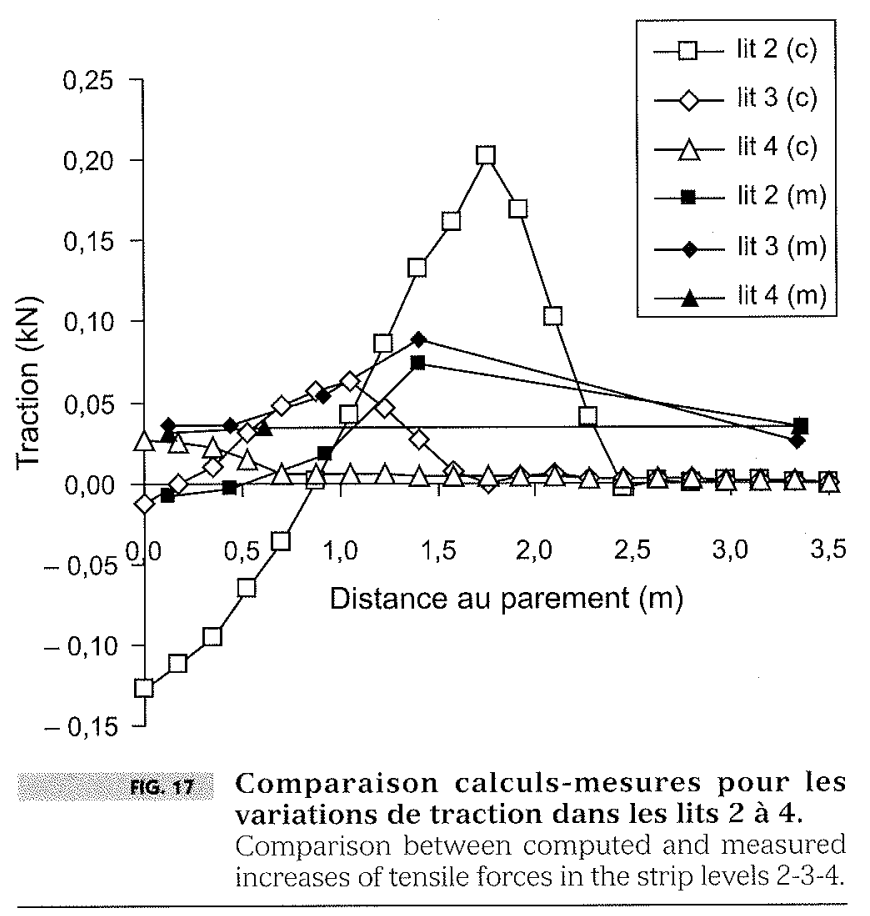

6

\section{Validation des interfaces par la modélisation d'un chargement plus important}

En complément de l'essai de chargement à $90 \mathrm{kN}$ réalisé pour le concours de prévision, un essai de chargement à $850 \mathrm{kN}$ a été entrepris en début d'année 2009. Le système de chargement a été adapté en conséquence. Le portique de réaction pèse $950 \mathrm{kN}$ et le vérin est capable d'appliquer, en statique, une force de 1000 kN (Fig. 18). La traverse a été remplacée par une superposition de plaques de répartition rectangulaires en acier de tailles différentes (Fig. 19). La plus grande mesure $0,9 \mathrm{~m}$ de large pour 2,4 $\mathrm{m}$ de long. Ĺimplantation sur l'ouvrage est la même que celle de la traverse.
Le modèle 3D (modèle multiphasique) permet d'avoir en un seul calcul les variations de traction dans les quatre lits d'armatures. Les mesures ont été réalisées à l'aide de jauges placées à proximité du mur : on n'a donc pas de valeur dans la partie droite des armatures, où se trouve l'augmentation maximale calculée (Fig. 20). L'ordre de grandeur des variations calculées avec le modèle 3D est correct. La différence que l'on peut observer pour le premier mètre d'armature, qui est d'ailleurs de moins en moins marquée avec la profondeur, est probablement à attribuer à un défaut de prise en compte du compactage lors de la simulation de la mise en ouvre. A cette étape, les tractions calculées sont moins importantes. On récupère, avec l'application de ce chargement important, les accumulations de traction générées dans la réalité lors du compactage réel.
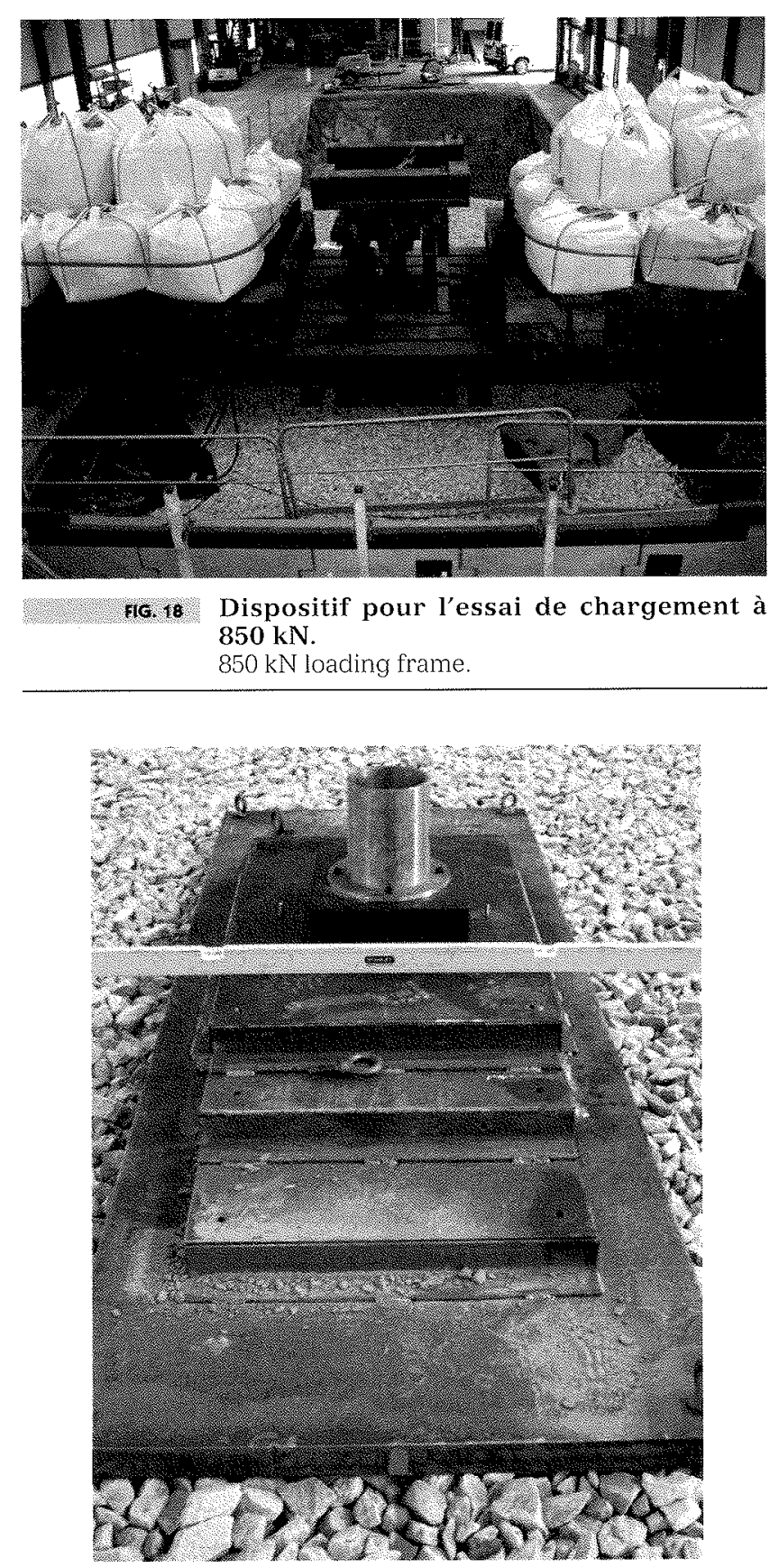

Fic. 19 Plaques de répartition.

Plates used to distribute the load over the ballast. 


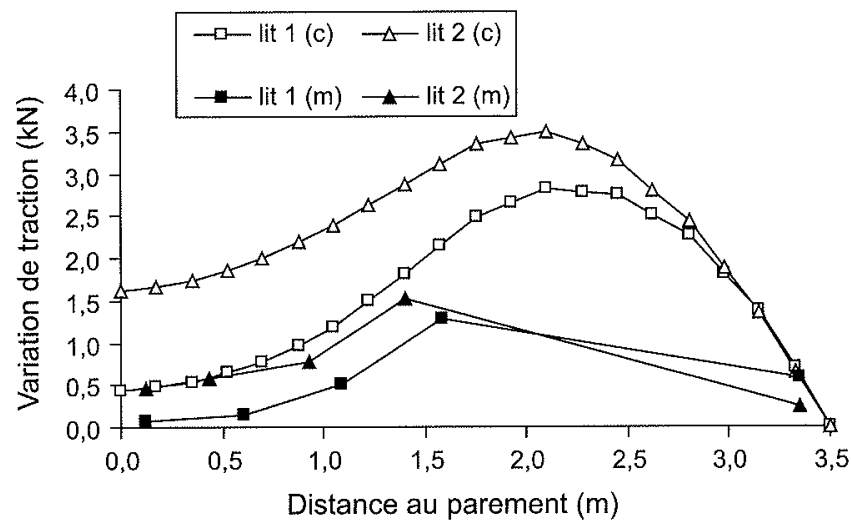

न. 20. Variations de tractions liées à l'application de la charge de $850 \mathrm{kN}$ mesurées (m) et calculées (c) dans les lits 1 et 2 avec le modèle $3 \mathrm{D}$.

Increase of tensile forces due to the $850 \mathrm{kN}$ loading measured $(\mathrm{m})$ and computed $(\mathrm{c})$ for $3 \mathrm{D}$ model in the strip levels 1 and 2 .

Le calcul 2D (modèle discret) mené avec la même méthodologie que pour le recalage sur les mesures sous un chargement de $90 \mathrm{kN}$ donne une estimation des variations de traction dans le lit 3 que l'on peut juger satisfaisante étant donné la rusticité du modèle (Fig. 21).

Les deux calculs donnent des déplacements verticaux de l'interface remblai/sous-couche qui sont en accord avec les mesures (Tableau II). Par contre, l'écart entre les tassements calculés aux points 1 (situé à $1,8 \mathrm{~m}$ du parement) et 2 (situé à $4,2 \mathrm{~m}$ du parement) est plus faible que l'écart entre les mesures.

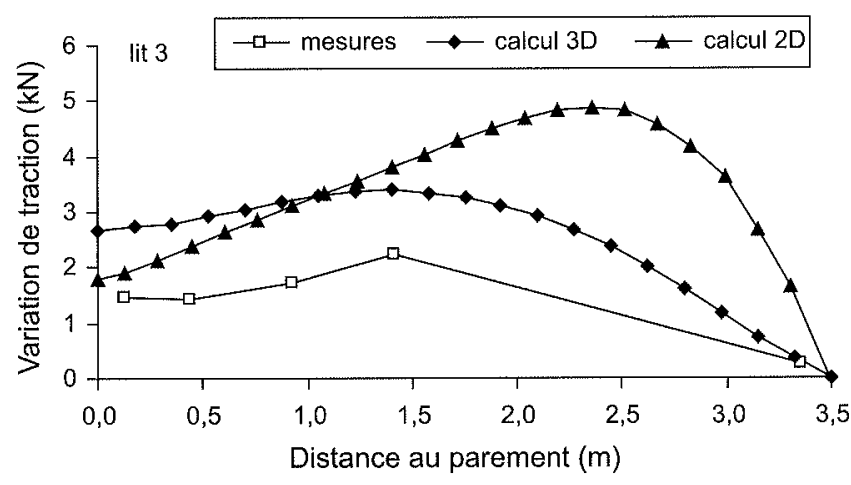

16. 21 Variations de tractions liées à l'application de la charge de $850 \mathrm{kN}$ mesurées et calculées dans le lit 3 avec les modèles 2D discret et 3D multiphasique.

Increase of tensile forces due to the $850 \mathrm{kN}$ loading measured and computed for 2D discrete and 3D multiphase models in the strip levels 1 and 2 .

Tartexull Comparaison des déplacements verticaux calculés et mesurés liés à l'application de la charge de $850 \mathrm{kN}$

Comparison between measured and computed vertical displacements due to the $850 \mathrm{kN}$ loading.

\begin{tabular}{|c|c|c|}
\hline Tessenent (IIIn) & pont & PONL2 \\
\hline Mesures & $-2,4$ & $-1,7$ \\
\hline Calcul 3D & $-2,2$ & $-2,1$ \\
\hline Calcul 2D & $-2,2$ & $-2,0$ \\
\hline
\end{tabular}

Enfin, les deux modèles permettent d'avoir une bonne estimation des déplacements horizontaux du parement (Fig. 22). Les courbes présentent des allures similaires et le maximum, atteint au tiers supérieur du parement, est obtenu à $30 \%$ près.

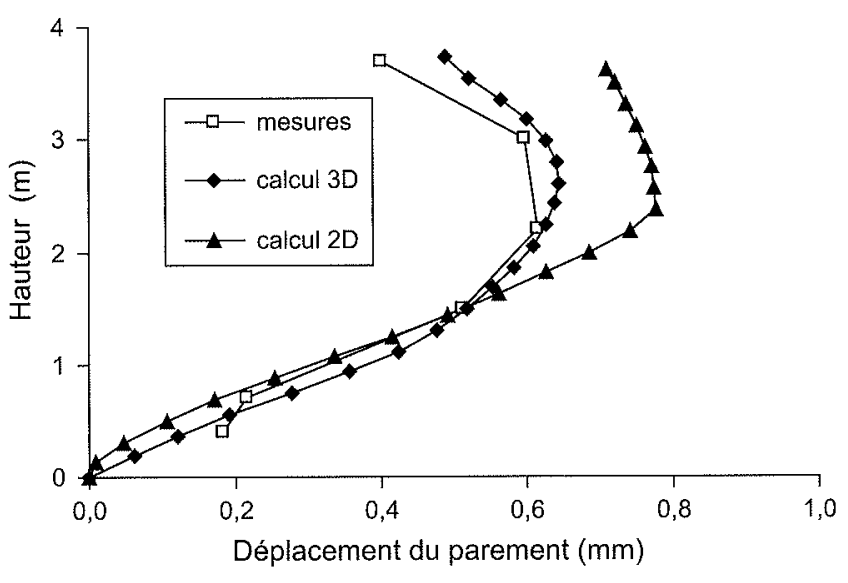

Ha. 22. Déplacements horizontaux du parement liés à l'application de la charge de $850 \mathrm{kN}$ mesurés et calculés avec les modèles 2D discret et 3D multiphasique.

Facing horizontal displacements due to the 850 $\mathrm{kN}$ loading measured and computed for 2D discrete and 3D multiphase models in the strip levels 1 and 2 .

\section{Conclusion}

Les différentes études paramétriques présentées ici montrent que l'on peut comprendre le comportement global du mur à l'aide du modèle multiphasique, à condition de prendre en compte le caractère tridimensionnel du problème, de bien choisir les caractéristiques mécaniques utilisées pour représenter la raideur de l'interaction entre le sol et les armatures et la valeur limite du frottement entre le sol et les armatures. Il est intéressant de noter que l'on a utilisé deux approches de modélisation dans lesquelles ces caractéristiques sont représentées par des paramètres entièrement différents, et que les deux approches fournissent finalement des résultats en bon accord, chacune nécessitant des ajustements particuliers.

La principale difficulté rencontrée dans la modélisation tient au compactage des couches de sol au cours de la construction. Les effets du compactage modifient les efforts dans les armatures, notamment au voisinage du parement. Il est donc difficile d'estimer et de prendre en compte les effets mécaniques dans les calculs. Des améliorations pourraient être envisagées en utilisant des lois de comportement plus complexes pour le sol, en modifiant les caractéristiques de résistance du remblai dans les différentes couches pour tenir compte des effets de compaction en profondeur.

\section{REMEREIEMENTS}

Les auteurs tiennent à remercier Réseau ferré de France et l'Association nationale de la recherche et de la technologie pour leur soutien financier. 


\section{Bibliographie}

AFNOR - Renforcements des sols. Ouvra ges en sols rapportés renforcés par armatures ou nappes peu extensibles. 1998.

Bastick M. - L'apport de la méthode des éléments finis à l'étude du comportement des ouvrages en Terre Armée. Journée d'étude sur l'utilisation des éléments finis, Paris, 1987, p. 257-263.

Benhamida B. - Modélisation numérique des murs en sol cloué : application aux calculs en déformations des murs expérimentaux en vraie grandeur $n^{\circ s} 1$ et 2 du projet national Clouterre. Thèse ENPC, 1998, $395 \mathrm{p}$.

Bennis M., de Buhan P. - A multiphase constitutive model of reinforced soils accounting for soil-inclusion interaction behaviour. Math. Comput. Modelling 37 2003, p. 469-475

Buhan (de) P. - Approche fondamentale du calcul à la rupture des ouvrages renforcés. Thèse dÉtat, université Paris 6 , 1986.
Buhan (de) P., Bourgeois E., Hassen G. Numerical simulation of bolt-supported tunnels by means of a multiphase model conceived as an improved homogenization procedure. Int. J. for Num. and Analytical Meth. in Geomechanics, 32 13, 2008, p. 1597-1615

Greuell E. - Étude du soutènement des tunnels par boulons passifs dans les sols et les roches tendres par une méthode d'homogénéisation. Thèse de l'École polytechnique, 1993

Mestat P., Berthelon J.-P. - Modélisation par éléments finis des essais sur fondations superficielles à Labenne, Bulletin des LPC n ${ }^{\circ} 234$, 2001, p. 57-78.

Richer S. - Résolution des problèmes de contact entre solides élastiques par la méthode des éléments finis. Thèse ENPC $1985,130 \mathrm{p}$

Rospars C., Bourgeois E., de Buhan P. Humbert P. - Modélisation numérique de la construction d'un mur expérimental en Terre Armée à Bourron Marlotte à l'aide d'un modèle homogénéisé. ColIoque Int. de Géotechnique, Beyrouth, 2004 , p. $275-280$

Sellali-Haraigue N. - Modélisation des contacts dans le calcul des ouvrages géotechniques. Thèse ENPC, 1999, 298 p.

Soyez L.. Le Kouby A. - Concours de prévision du comportement d'un mur en terre armée en vraie grandeur. Revue française de géotechnique, $n^{\circ}$ 129, 2009

Sudret B., de Buhan P. - Multiphase mode for inclusion-reinforced geostructures. Application to rock-bolted tunnels and piled raft foundations. Int. J. Numer. Anal. Meth. Geomech, 25, 2001, p. 155182.

Unterreiner P., Benhamida B., Schlosser F. - Finite element modelling of the construction of a full scale experimental soil nailed wall. Int. $J$. Ground Improvement, vol 1, n¹, 1997, p. 1-8. 